\title{
Archéopages
}

Archéopages

Archéologie et société

Hors-série 3 | 2012

Nouveaux champs de la recherche archéologique

\section{Prologue. La vocation scientifique de l'Inrap}

Jean-Paul Demoule et Jean-Paul Jacob

\section{OpenEdition}

Journals

Édition électronique

URL : https://journals.openedition.org/archeopages/441

DOI : 10.4000/archeopages.441

ISSN : 2269-9872

\section{Éditeur}

INRAP - Institut national de recherches archéologiques préventives

\section{Édition imprimée}

Date de publication : 1 janvier 2012

Pagination : 4-13

ISSN : 1622-8545

\section{Référence électronique}

Jean-Paul Demoule et Jean-Paul Jacob, «Prologue. La vocation scientifique de l'Inrap », Archéopages

[En ligne], Hors-série 3 | 2012, mis en ligne le 01 janvier 2012, consulté le 25 février 2023. URL : http:// journals.openedition.org/archeopages/441 ; DOI : https://doi.org/10.4000/archeopages.441 

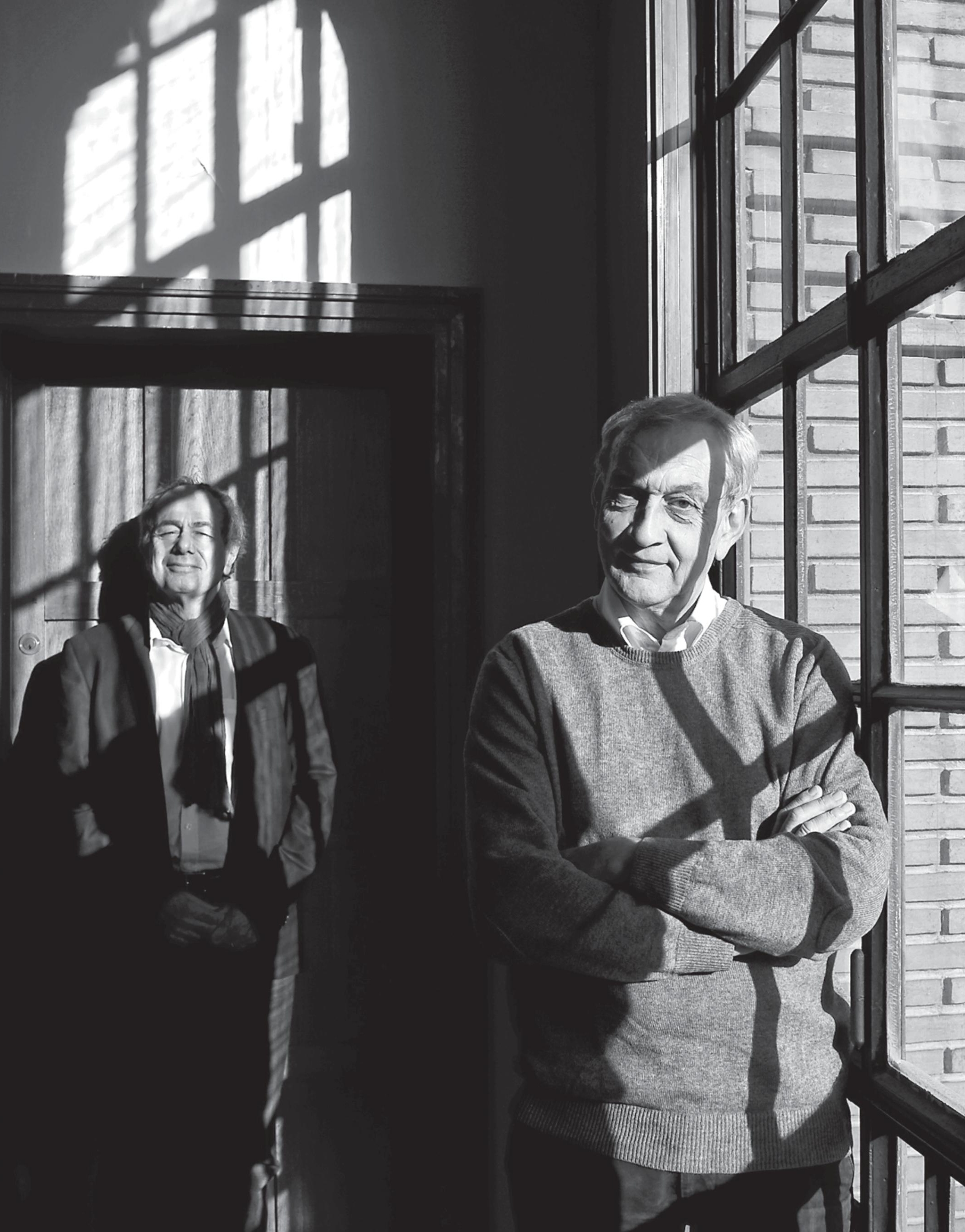


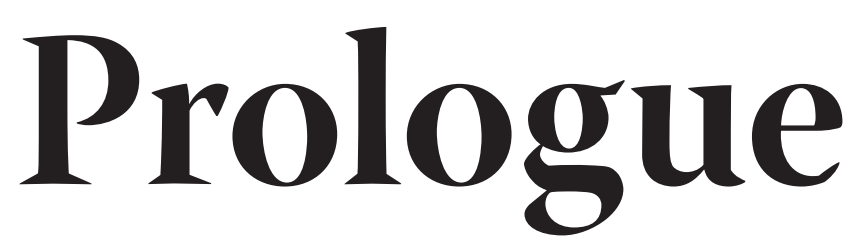

La vocation scientifique de l'Inrap

Jean-Paul Demoule

Professeur à l'Institut universitaire de France

Président de l'Inrap de 2002 à 2008

Jean-Paul Jacob

Président de l'Inrap

Ce numéro spécial consacré aux nouveaux champs de la recherche pour les 10ans de l'Inrap a fourni l'occasion, en février 2012, d'un débat sur la vocation scientifique de l'Institut, sa construction, ses enjeux et ses résultats. 


\section{Prologue}

\section{La vocation scientifique de l'Inrap}

\section{Le projet d'un institut national de recherches archéologiques préventives}

JPJ Dans les années 1970 et 1980, il y avait une confusion totale entre les services de l'État et l'Afan, à telle enseigne que le président de l'association était le sous-directeur de l'archéologie au ministère de la Culture et que les conservateurs régionaux de l'archéologie, alors encore appelés les directeurs des antiquités, avaient les chéquiers de l'association... Sur le plan juridique, cela constituait une situation de «gestion de fait». En outre, sur le plan scientifique, cela plaçait les archéologues de l'Afan dans une position assez ancillaire vis-à-vis des conservateurs régionaux. C'est pourquoi s'est posée, de façon récurrente, la question de la forme juridique et organisationnelle à donner à l'association et que j’ai toujours été mû par le souci qu'un régime juridique digne de ce nom soit donné à l'archéologie que l'on disait à l'époque encore «de sauvetage». C'est d'ailleurs pour cette raison que j'ai quitté le CNRS en 1982 pour le ministère de la Culture, afin d'être au plus près des enjeux de terrain.

JPD Pour ma part, j'ai toujours été frappé par le fait que la France a créé l'École française d'Athènes en 1846, l'École française de Rome en 1872, ou l'Institut français d'archéologie orientale en 1880, mais que, à la fin des années 1990, plus d'un siècle plus tard, notre pays n'avait toujours pas d'institut de recherche dévolu à l'archéologie de son propre territoire, pourtant dite «nationale», le Centre de recherche archéologique du CNRS n'ayant été qu'une expérience sans lendemain, et non spécifiquement métropolitaine. C'était un paradoxe sur le plan scientifique qui a des racines assez profondes dans l'histoire de la pensée politique en France... Et depuis le début de mon activité d'archéologue dans les années 1970, notamment dans la vallée de l'Aisne, la question de la création d'un institut de recherche m’a préoccupé. Elle a pris un relief particulier avec le développement de l'archéologie de sauvetage, qui allait devenir l'archéologie préventive. Le volume des travaux, et les effectifs impliqués avaient considérablement augmenté et la discipline est allée de crise en crise. De fait, l'Afan était devenue la deuxième association en France en termes d'effectifs. Le débat qui a couru au fil des nombreux rapports sur l'archéologie - de 1974 à 1998 -, était de déterminer quelle serait la structure la plus adaptée : un établissement public industriel et commercial, pour recevoir les fonds des aménageurs, ou un établissement public administratif, proche dans sa structure de celle des établissements de recherche. Lors de la crise de 1998, j'ai été chargé d'une mission avec Bernard Pêcheur, un conseiller d'État, ancien directeur de la Fonction publique, et Bernard Poignant, le député-maire de Quimper. Nous avons rencontré des représentants de toutes les professions archéologiques et s'est imposée l'idée que si l'on créait une structure, il valait mieux que cela soit un institut de recherche avec des missions assez larges.
JPJ Pour ma part, j'étais partisan de l'intégration d'une partie des agents de l'Afan au sein du ministère de la Culture pour faire ce que l'on appelait à l'époque les sondages et que sont aujourd'hui les diagnostics, dans la mesure où il s'agit de la partie régalienne de l'activité archéologique qui fonde la prescription. Mais je nétais malgré tout pas tout à fait convaincu de cette option car je pense qu'un bon prescripteur doit être un bon fouilleur et qu'un bon fouilleur sera un bon diagnostiqueur. In fine, c'est le scientifique qui a prévalu sur la pure logique juridique et c'est tant mieux. JPD De fait, en 1985, la première vague d'intégration de ces archéologues de l'Afan s'est faite au sein de la sous-direction de l'archéologie où, sur 250 agents, la moitié vient de l'Afan, parmi lesquels de nombreux conservateurs régionaux aujourd'hui en poste.

C'était, dix-sept ans avant la création de l'Inrap, une première forme de reconnaissance des compétences scientifiques des archéologues de l'Afan.

JPJ Comme conservateur régional de l'archéologie de 1981 à 1996, je me suis battu dès les années 1980 pour ne plus être seul signataire des notices des informations de Gallia, estimant que le fouilleur devait signer aussi, et non pas le seul conservateur régional ; c'était une reconnaissance légitime de la qualité scientifique des personnes titulaires de l'autorisation de fouilles, qu'elles soient de l'Afan ou même bénévoles.

JPD Pour la plupart d'entre nous, toutes institutions confondues, il est évident que la fouille est un acte scientifique puisque c'est la façon dont on fouille qui conditionne le reste de la recherche : pour prendre un seul exemple, si on relève un trou de poteau sans savoir quelle structure on peut attendre, on ne va pas trouver cette structure... Donc il n'y aurait pas dû y avoir de débat sur le statut de l'Inrap. Mais, dans différents ministères et dans les avis du Conseil de la Concurrence en 1998, prévalait la dichotomie entre deux archéologies sans rapport l'une avec l'autre : une archéologie "de sauvetage », qui aurait relevé d'une activité de terrassement, et une archéologie considérée comme de la «vraie science». Pourtant, personne n'estime que, lorsqu'un chercheur du CNRS fouille sa truelle à la main, c'est un terrassier, alors que lorsqu'il est derrière son ordinateur, c'est un vrai chercheur ! Tout cela est évidemment intégré...

Lorsque nous avons interrogé la profession, Bernard Pêcheur et Bernard Poignant ont évidemment compris que l'archéologie est une démarche globale, de la fouille à l'exploitation scientifique des résultats. Il était donc rationnel de proposer la transformation de l'Afan en un institut de recherche. D'autant plus qu'il fallait exploiter scientifiquement les résultats de fouilles préventives innombrables, ce que ne pouvaient plus faire les seuls archéologues des services régionaux, du CNRS ou de l'université. Enfin, il y avait la question de la restitution des résultats au public, qui apparaissait comme un enjeu majeur de diffusion culturelle et une nécessité impérieuse pour rendre la discipline légitime aux yeux de tous. JPJ Le fait d'opter pour un institut national tenait aussi compte de l'évolution des compétences. Au fil des années, un certain nombre d'archéologues recrutés comme techniciens étaient devenus des chercheurs de haut niveau. Une agence de moyens, sous statut 
associatif ou sous un autre statut, n'aurait pas permis de faire durablement évoluer les compétences de cet extraordinaire vivier de chercheurs et de très bons techniciens qu'était devenue l'Afan. Avec dix années de recul, c'est d'une évidence implacable. Nous le mesurons clairement à travers l'implication des archéologues de l'Inrap dans les structures collectives de recherche.

\section{La recherche à l'Afan}

JPJ Au début, les agents de l'Afan prenaient souvent sur leur temps personnel - qui était en général rémunéré par l'Unedic... - pour faire de la recherche après la fouille et même parfois pour rédiger le rapport. Mais il n'y avait pas de recherche organisée et c'étaient les services régionaux de l'archéologie qui, dans le meilleur des cas, incitaient et coordonnaient les publications. Cela étant, les archéologues de l'Afan n'étaient pas suffisamment considérés, et l'on trouve encore aujourd'hui des chercheurs qui estiment que ceux de l'Inrap sont une catégorie à part... JPD C'est ridicule, d'autant que, je le disais, aujourd'hui le CNRS n'aurait pas les forces pour exploiter le volume considérable de données qu’apporte l'archéologie préventive. Mais, en outre, peu de ses chercheurs en auraient les compétences : moi-même, aujourd'hui, je serais incapable de conduire une fouille préventive de grande surface, urbaine en tout cas...

\section{L'organisation de la recherche à l'Inrap}

JPD La première décision forte a été la création d'une direction scientifique, explicitement mentionnée dans le décret statutaire. Avec JeanPierre Giraud, le premier directeur scientifique, qui venait du ministère de la Culture, puis avec Pascal Depaepe son successeur, issu de l'Inrap, nous avons défini des programmes de recherche en nous appuyant sur un conseil scientifique, prévu par le décret statutaire. Dans le même temps, nous avons fait émerger des projets scientifiques, en leur attribuant des moyens spécifiquement dévolus . De «mon temps», on n'en avait pas assez, mais c'était déjà un progrès considérable.

JPJ Actuellement, l'Inrap consacre 18600 jourshomme à la recherche (entendue, par commodité, comme l'ensemble des activités qui se font au-delà de la remise du rapport de fouille). Sur ce quota sont «financées » toute une série de participations à des instances scientifiques, qui sont indispensables mais qui ne sont pas de la recherche à proprement parler. Donc, seuls 13 ooo à 14 ooo jours sont effectivement consacrés à la recherche, et notamment aux projets d'activité scientifique (PAS). Cela représente un ratio d'environ 6 à $7 \%$ de l'activité. Ce chiffre n'a pas évolué en valeur absolue malgré la progression des effectifs en CDI, constante sur la période, et c'est évidemment insuffisant.

JPD Dans les années 2002 à 2008, on pouvait satisfaire environ un tiers des demandes émanant des agents de l'Inrap. Où en êtes-vous maintenant? JPJ Actuellement, avec l'aide du conseil scientifique, ce sont environ $50 \%$ des demandes qui ne peuvent être retenues. Si nous sommes en progrès, le niveau de l'enveloppe reste insuffisant pour accompagner les projets des agents qui légitimement souhaitent développer leurs recherches, dans le cadre de la programmation scientifique de l'institut et la définition d'axes de recherches, à travers les projets d'activités scientifiques, et au sein des unités mixtes de recherche. Par ailleurs, l'ambition doit être d'accroître le soutien aux jeunes chercheurs, et notamment les thésards, ce qui se fait chaque année mais on pourrait encore améliorer les choses. Enfin, je mène avec Arnaud Roffignon, le directeur général, des démarches pour que l'Inrap soit reconnu comme « organisme de recherche» par le ministère de l'Enseignement Supérieur et de la Recherche, et nous avons désormais bon espoir d'y parvenir, avec l'aide du ministère de la Culture.

\section{Les axes de recherche}

JPD La définition d'axes de recherche était indispensable pour ne pas éparpiller les moyens et pour concentrer les efforts sur les problématiques les plus importantes, les questions nouvelles, les synthèses... Par ailleurs, personnellement, j'estime que le Conseil national de la recherche archéologique ne définit pas suffisamment de priorités, notamment dans le champ de l'archéologie préventive où le nombre d'opérations est très important et où les choix pourraient être guidés par des orientations nationales. Il était donc indispensable que l'Inrap définisse ses propres axes de recherche.

JPJ L'un des atouts de l'institut, c'est d'avoir une vision nationale des travaux de recherche, pour l'ensemble des périodes et des domaines. Par essence tête de réseau et lieu de synthèse, l'Inrap est certainement l'une des seules structures en France à être capable de conduire de grands travaux de synthèse comme l'enquête nationale sur lâge du Bronze et le premier âge du Fer, ou celle sur le second âge du Fer. Pascal Depaepe conduit aussi un chantier qui contribue à mieux corréler les données sur le Paléolithique, celles du nord comme celles du sud. Cela semble simple, mais n'avait pas été fait. C'est tout à fait fondamental d'exploiter des centaines de milliers de données émanant de milliers de sites. Nous voyons ainsi les lacunes et pouvons contribuer à faire émerger une nouvelle génération de chercheurs, comme dans le domaine de l'Antiquité gallo-romaine, qui est, toutes institutions confondues, un peu sinistrée actuellement.

JPD - En effet, si l'on observe l'état de la recherche par grandes périodes, la situation est satisfaisante pour la Préhistoire et la Protohistoire. En ce qui concerne Moyen Âge, la recherche connait un développement important, et d'ailleurs les historiens commencent à prendre en compte nos travaux. Mais, pour la période gallo-romaine, il y a un hiatus entre ce que l'Inrap apporte en termes de connaissance des installations rurales ou des agglomérations secondaires, et la recherche académique qui reste attachée à des problématiques traditionnelles, que les données de l'archéologie préventive rendent un peu «archaïques».

\section{L'émergence des problématiques}

JPJ Il y a un aller et retour permanent entre les questions que l'on se pose $a$ priori et les données qui remontent du terrain. Mais la spécificité de 
l'archéologie, c'est évidemment de travailler sur des données matérielles, sans a priori. Il est certain, par exemple, que la fouille systématique de grandes surfaces et le développement de l'archéologie urbaine ont imposé de nouvelles approches : sur les fermes indigènes, sur l'époque paléochrétienne, sur le haut Moyen Âge... Et à partir des données, on construit des modèles, que l'on remet en cause au fil des découvertes et de l'évolution des méthodes, qui progressent de façon spectaculaire comme l’a montré le récent colloque «L'Archéologie au laboratoire» que l'Inrap a coproduit avec le département R\&D d'EDF'.

JPD Les grands changements ont été parfois suscités par l'archéologie préventive et se sont étendus à l'ensemble de la communauté scientifique. Ainsi, les résultats de fouilles de grandes surfaces ont motivé la reprise de fouilles programmées sur des thèmes renouvelés. Personnellement, ce qui me paraît le plus fondamental ce sont les approches territoriales: il ne s'agit plus de fouiller un site prestigieux isolé mais de comprendre l'ensemble du fonctionnement d'un territoire.

\section{L'enjeu d'un institut unique}

JPD Dans le système anglo-saxon, la recherche se fait à l'université. En France, on est plutôt accoutumés à avoir des instituts nationaux. Bénéficier d'une structure nationale permet de réconcilier deux traditions longtemps « étanches » l'une à l'autre - l'archéologie préhistorique et l'archéologie historiqueet de faire émerger des problématiques transversales. JPJ L'histoire est un continuum : avoir un institut qui couvre toute les périodes, à l'échelle nationale, est extrêmement important. C'est pour cela que j'ai proposé que la programmation scientifique de l'institut, qui est arrêtée après avis du conseil scientifique, puisse être présentée au Conseil national de la recherche archéologique et ainsi s'intégrer dans une réflexion encore plus globale sur la politique archéologique.

JPD Cette unité de la recherche, permise par l'Inrap, est d'autant plus importante que l'organisation récente du CNRS a réinstauré la vieille césure épistémologique du XIX ${ }^{\mathrm{e}}$ siècle entre études préhistoriques, aujourd'hui rattachées à l'INEE, et archéologie historique placée dans l'orbite de l'INSHS. JPJ Lune des richesses de l'Inrap, outre la couverture complète du champ chronologique, c'est la pluridisciplinarité. Il n'y a pas d'autre exemple, à cette échelle, dans le domaine de l'archéologie, d'institution pluridisciplinaire. C'est un modèle très original, d'une grande exigence scientifique et méthodologique, qui permet d'intégrer l'ensemble des problématiques dès la fouille, et de mobiliser «en temps réel» tout le spectre des compétences. JPD Un des atouts d'une structure d'une certaine taille, c'est aussi qu'elle peut se doter d'outils de diffusion des résultats scientifiques - comme cette revue même, le site internet ...- ou se doter de compétences de valorisation, qui manquaient jusque-là dans le champ de l'archéologie - comme la production audiovisuelle documentaire. L'Inrap a ainsi pu mettre en œuvre une politique publique de diffusion de la connaissance archéologique -

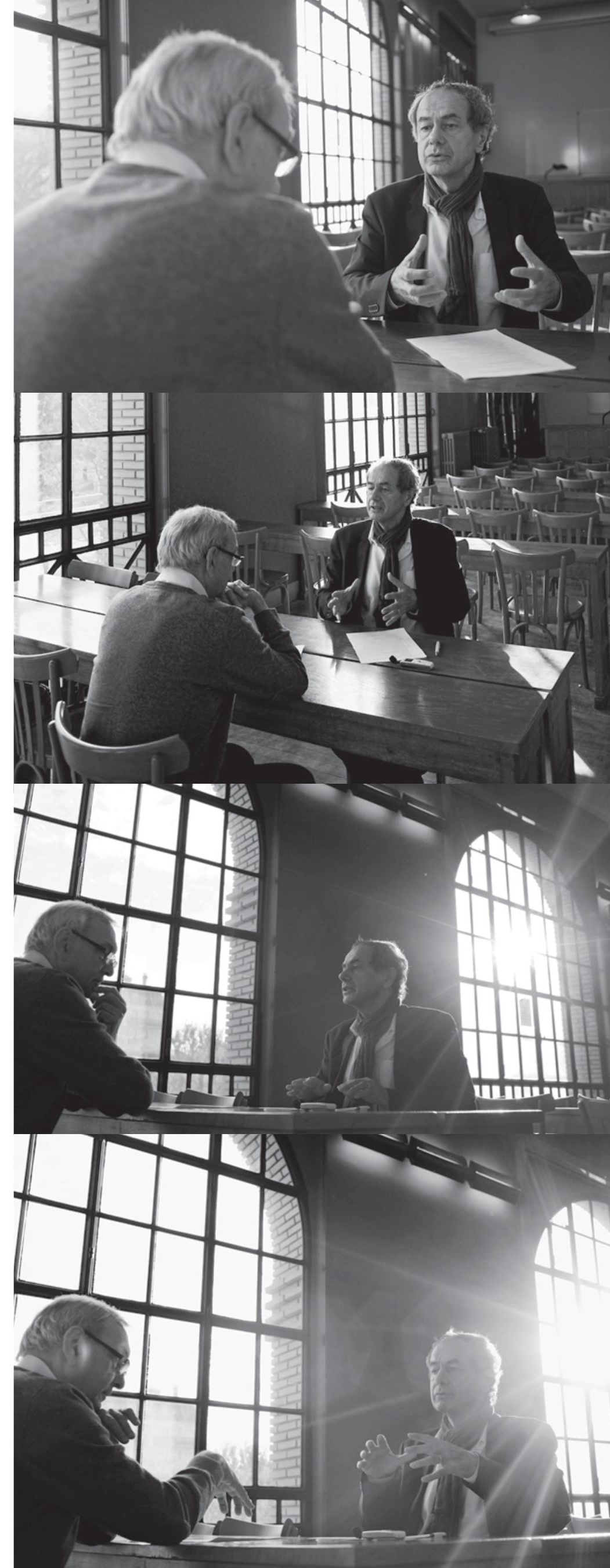


à l'instar du Louvre dans le champ de l'histoire de l'art - qui est indispensable pour faire accepter les contraintes de l'archéologie préventive, mais qui, surtout, manquait dans notre pays en termes de diffusion culturelle.

\section{Le choix des projets}

JPD On est passé des «projets individuels de recherche » aux "projets d'action scientifique » qui se sont intégrés, à quelques exceptions près, dans les axes de recherche de l'Inrap et qui font la part belle à des travaux d'équipe. Il y a eu un encouragement très important aux publications, ainsi qu'à la mise en ligne des rapports de fouilles, qui sortent du statut de littérature grise et sont ainsi accessibles à l'ensemble de la communauté scientifique (et au-delà).

\section{L'évaluation scientifique}

JPJ L'évolution des projets de recherche est aussi liée à celle des compétences scientifiques : or on assiste à une progression régulière du niveau scientifique des archéologues de l'Inrap ; ainsi, aujourd'hui 130 agents sont docteurs, dont quelques un HDR et plusieurs centaines sont au moins d'un niveau master. Un des autres atouts de l'institut c'est l'émergence de spécialistes de plus en plus pointus, reconnus par leurs pairs. L'ensemble de ces personnels doit pouvoir bénéficier d'une évaluation scientifique, ce qui confortera cette reconnaissance. $\mathrm{Et}$, comme je l'indiquais tout à l'heure, l'institut devra aussi être évalué par l'Agence d'évaluation de la recherche et de l'enseignement supérieur (AERES). JPD De mon point de vue, cette évaluation est capitale. Elle confortera le statut scientifique de l'Inrap. Comment envisagez-vous sa mise en œuvre? JPJ Il s'agit d'un chantier important de l'année 2012 et nous n'avons pas encore arrêté le dispositif dans toutes ses modalités. Mais ce que je peux dire, c'est que, pour les archéologues de l'Inrap, elle sera concentrée sur les agents de catégories quatre et cinq. Les agents de catégorie trois et deux pourraient être également évalués, sur la base du volontariat. L'enjeu est de déterminer un dispositif à la fois simple et efficace qui puisse bien évidemment reposer sur une évaluation par les pairs. Nous avons pour objectif de mettre en place le dispositif à compter de 2013. JPD Il faudrait faire comme au CNRS et avoir une catégorie d'ingénieurs-techniciens d'une part, et de l'autre une catégorie de chercheurs fortement impliqués. Cette question est restée en jachère de 2002 à 2008, car, avec Marion Julien puis Nicole Pot - les deux directrices générales avec lesquelles j'ai eu l'honneur de travailler - il y avait plus urgent. Mais je pense maintenant que cela n'aurait rien de dévalorisant et qu'il faudrait deux catégories distinctes...

JPJ Beaucoup a été fait, mais il y a encore tant à faire, je peux l'assurer! Par ailleurs, il ne faut pas confondre l'évaluation scientifique des agents avec l'évaluation de leur «manière de servir », qui est une obligation réglementaire et s'applique à l'ensemble des agents - nous venons de la mettre en place pour la filière administrative, et elle sera étendue à la filière scientifique et technique en 2013, avec l'évaluation scientifique. En effet, les temps ne peuvent être les mêmes et si l'entretien professionnel doit être annuel, l'évaluation scientifique ne prend de sens que dans un temps plus long.

JPD Si l'on prend le CNRS, ses ingénieurstechniciens sont évalués par le patron du laboratoire suivant leurs compétences, et les chercheurs sont évalués par des commissions scientifiques.

JPJ Pour l'évaluation scientifique, il faut que l'Inrap fasse comme d'autres structures. Il faut réfléchir à un système de commissions, en lien avec le conseil scientifique. De fait, comme au CNRS, ce sont les pairs qui évalueront. Et cette évaluation doit pouvoir s'appuyer notamment sur les publications, la participation active à des équipes de recherche, la participation aux colloques, etc.

\section{Les moyens de la recherche}

JPJ Aujourd'hui, nous l'avons dit, les moyens dévolus à la recherche sont insuffisants. 18600 jours-homme, ce n'est pas assez. Il n'en reste pas moins que l'Inrap offre des possibilités que l'on ne trouve nulle part ailleurs lorsque l'on est archéologue pour allier terrain et recherche. Beaucoup de jeunes chercheurs très prometteurs l'ont bien compris. Avec la réforme de la redevance d'archéologie préventive (RAP) que le Parlement vient d'adopter, les moyens alloués à l'archéologie préventive ont été consolidés et nous ne dépendrons plus de subventions «exceptionnelles» mais qui étaient devenus hélas récurrentes. La réforme ne permettra a priori toutefois pas de consacrer plus de moyens à la recherche, l'ambition du gouvernement n'ayant pas rencontré le consensus au niveau du Parlement. On peut toutefois espérer que le montant de la RAP soit augmenté dans les années à venir, le dispositif actuel constituant un socle solide. Par ailleurs, la part de la RAP qui finance la recherche est enviée par les services des collectivités territoriales, voire par les opérateurs privés et, là, on touche à un problème de fond car la loi de 2003 n'est pas une loi de décentralisation ; le législateur a bien confié à l'institut un rôle spécifique d'exploitation et de valorisation de l'ensemble des résultats, bien au-delà de la libération des terrains. L'Inrap, je le disais précédemment, a un rôle de synthèse et les moyens ne peuvent être dispersés si l'on conserve cette ambition. Mais l'Inrap est aussi un lieu de débat et l'ensemble des contributions à la recherche, quels qu'en soient les acteurs, doit pouvoir être discuté et confronté. C'est pourquoi l'institut a développé une large politique de partenariat avec nombre d'institutions, bien sûr avec le CNRS mais également avec les services agréés de collectivités territoriales. JPD Un rôle spécifique d'exploitation et de valorisation de l'ensemble des résultats, c'est bien la vocation de l'établissement. Cependant, jusqu'à présent, le ministère de l'Enseignement Supérieur et de la Recherche n'a pas financé l'Inrap. Il serait logique qu'il y contribue à hauteur de 20 à 30 millions d'euros environ. Il finance bien la Cité des sciences et de l'industrie ou le musée du quai Branly qui ne sont pas des institutions plus scientifiques que l'Inrap. Cela pourrait être une source de financement supplémentaire, exclusivement fléchée à la Cité des Sciences

et de l'Industrie. Actes

à paraître en janvier 2013

Stéphanie Thiébault

et Pascal Depaepe,

coédition Inrap/L

Découverte 
sur la recherche, indépendamment des diagnostics ou des fouilles.

JPJ Je le pense aussi et j'ai déjà eu l'occasion de le dire à plusieurs reprises à nos interlocuteurs. Puisse cette demande être exaucée un jour!

\section{Les collaborations scientifiques}

JPD En 2002, nous avions lancé avec la sousdirection de l'archéologie et le CNRS, les activités collectives de recherche (ACR), qui réunissaient les différentes institutions de recherche et étaient assorties d'aides financières pour encourager un certain nombre de projets pluri-institutionnels. Où en êtes-vous?

JPJ Nous venons de signer une nouvelle convention- cadre avec le CNRS, qui prévoit des échanges de chercheurs entre le CNRS et l'Inrap, de façon à faciliter la porosité entre nos institutions. On pourra avoir des archéologues du CNRS sur nos fouilles et des archéologues de l'Inrap sur des chantiers CNRS. Nous sommes très présents dans 25 unités mixtes de recherche, et dans une vingtaine universités, parfois avec une très forte implication dans la formation des étudiants. Par ailleurs, actuellement, 7 archéologues de l'Inrap travaillent dans des SRA et nous aimerions que des archéologues de l'État puissent venir à l'Inrap; en dehors de périodes de stages. Évidemment, ceci en chagrine quelques-uns qui croient, bien à tort, que l'Inrap est alors juge et partie. Sans compter des conventions scientifiques et culturelles avec le musée du Louvre, le musée du quai Branly, la Cité de l'architecture et du patrimoine, l'École du Louvre et l'École du patrimoine par exemple. Un des objectifs de ces conventions est notamment de mieux former les étudiants à l'archéologie préventive, quelle que soit leur fonction ensuite. Nous avons besoin de partenaires qualifiés à l'avenir pour pouvoir faire de la recherche dans de bonnes conditions.

\section{Les enquêtes nationales}

JPJ Les enquêtes nationales rassemblent la communauté scientifique, pour réunir et synthétiser l'ensemble de l'information autour d'un thème. Cela permet de faire collaborer des chercheurs parfois très éloignés géographiquement et de faire se rencontrer des traditions qui s'ignorent. C'est un des outils majeurs dont nous nous sommes dotés pour mettre en œuvre nos missions de recherche.

\section{Les collaborations internationales}

\section{JPD À partir de 2002, nous avons noué des} relations internationales avec un certain nombre de pays pour favoriser les échanges de savoir-faire, les accueils de chercheurs, en France notamment, avec le Cambodge, l'Algérie, le Maroc, la Chine, la Russie, l'Albanie... car l'expérience et les méthodes de l'Inrap y sont enviées.

JPJ En effet, partout où je suis allé à létranger, linstitut est connu et reconnu ${ }^{2}$. Nous sommes de plus en plus sollicités. Ce qui est important, sur le plan de la recherche, outre le fait de mieux connaitre les sites où nous intervenons, c'est aussi de croiser les pratiques et de faire bénéficier les archéologues de l'Inrap d'expériences et de compétences nouvelles, dans des champs différents de ceux de l'archéologie nationale, avec des effets très féconds en retour pour nos recherches en France. C'est particulièrement vrai pour le Paléolithique ou le Néolithique. On peut citer par exemple la réception de travaux de Vincent Mourre sur la retouche par pression à Blombos Cave ou ceux de Laurent Bruxelles à Sterkfontein, en Afrique du Sud.

\section{Le développement de la concurrence}

JPD Ce qui est envié en Italie ou en Espagne, par exemple, c'est le fait que l'Inrap soit un institut national, qui déploie une politique de diffusion et de mise en relation des données. En Espagne notamment - qui a signé la convention de Malte -, les fouilles se déroulent mais les données ne sont mêmes pas connues de la communauté archéologique; on y a souvent affaire à une archéologie «alibi», qui a pour seule fonction de libérer les terrains. JPJ De surcroît, en Espagne, il y a un tas de petites structures qui font faillite à cause de la crise, ce qui induit une perte considérable de compétences et de données. À telle enseigne que le gouvernement espagnol envisage la création d'un institut national pour corriger ce désastre. Quant à l'archéologie préventive en Grande-Bretagne, où règne le libéralisme le plus dérégulé, elle est complètement sinistrée!

JPD De fait, plusieurs centaines d'archéologues anglais d'entreprises privées se sont retrouvés au chômage : cela pose non seulement des problèmes sociaux mais cela a aussi de graves conséquences scientifiques, car la documentation des sites est éparpillée, sinon définitivement perdue. Et dans les pays où il $y$ a différents intervenants, notamment en entreprises privées, ce ne sont pas forcément les meilleurs spécialistes qui sont choisis pour fouiller dans une région donnée : certains sites sont fouillés successivement, par des intervenants différents, avec une incohérence dans les méthodes et la documentation aboutissant à une absence de publication.

JPJ Dans le même ordre d'idées, on peut hélas citer le cloître Saint-Trophime en Arles, où l'Inrap a fait un diagnostic, une expertise scientifique détaillée à la demande du SRA. On y a mis au jour une galerie complète et un quart du jardin du cloître. Il y a eu un appel d'offres pour la fouille proprement dite et c'est un autre opérateur qui a été choisi. Juridiquement, c'est inattaquable, mais où est la cohérence scientifique? On a assisté à peu près à la même situation avec la synagogue médiévale de Montpellier.

JPD Normalement, la loi prévoit que l'Inrap soit destinataire de tous les rapports de fouille, qu'ils émanent des services de collectivités territoriales ou des opérateurs privés, précisément pour permettre les travaux de synthèse et la diffusion des données scientifiques.

JPJ La loi le prévoit et nous nous y attelons mais, dans la pratique, il est très difficile d'obtenir ces documents et cela obère gravement le développement de la recherche. Il faut rappeler que l'Inrap n'est pas un opérateur parmi d'autres et que la loi lui a confié d'autres missions que celle de réaliser des fouilles. JPD En situation de concurrence, il n'est pas surprenant que, les opérateurs rechignent à faire circuler les rapports de fouille... Précisément pour
2 Cf Archéopages

«Archéologie

sans frontières ",

hors-série $n^{\circ} 2$,

octobre 2010

3 Cf Archaeology and the

Global Economic Crisis.

Multiple Impacts,

Possible Solutions,

Nathan Schlanger et

Kenneth Aitchison (éd.),

ACE/Culture Lab

Éditions, 2010. http://ace.

archaeology.eu/

fichiers/25Archaeology-

and-the-crisis.pdf

4 Gaulois, une expo

renversante, 19 octobre

2011- 2 septembre 2012.

Qui étaient les Gaulois?

sous la direction de

François Malrain et

Mathieu Poux, catalogue

de l'exposition, coéditions

La Martinière/

Universcience

éditions, 2011

5 Nouveaux moyens,

nouveaux financements,

nouvelles problématiques en archéologie,

Conférences du Collège

de France - Colloque

"La Mondialisation

de la recherche", http://

conferences-cdf.revues.

org./329 


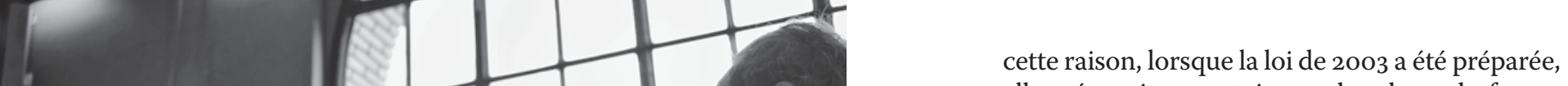

elle prévoyait un certain nombre de garde-fous pour éviter le risque d'éparpillement de l'information. Il faut que l'État les mette en œuvre. En outre, la concurrence ne peut fonctionner correctement que s'il y a un contrôle de la qualité scientifique et des méthodes, mais aujourd'hui les services régionaux de l'archéologie sont trop squelettiques, situation que tu dénonces toi aussi, pour être suffisamment efficaces. J'ai d'ailleurs un peu de mal à comprendre le jeu que jouent certaines DRAC avec la concurrence, encouragées, parfois, il est vrai, par les préfets. Cela pouvait se comprendre au début de l'application de la loi de 2003, mais certaines postures sont aujourd'hui particulièrement nuisibles non seulement pour l'Inrap mais pour l'archéologie en général. Ainsi, on a constaté que des agréments étaient donnés sans toutes les garanties nécessaires, que des opérateurs étaient retenus alors que le responsable d'opération dirigeait deux fouilles en parallèle ou, plus grave encore, que des fouilles étaient massacrées sur le plan méthodologique... Il y a là un sérieux problème, probablement en partie structurel, sur lequel j'ai eu l'occasion de m'exprimer.

JPJ Pour ma part, il me semble nécessaire que la concurrence soit loyale, ce qui n'est pas toujours, hélas, le cas, les standards d'exigence scientifique, la structure des coûts et les pratiques sociales n'étant pas identiques. Parfois, même, on note que ne sont pas répercutés dans les devis des coûts pris en charge par d'autres structures... Cela pose la question de la régulation, au sens économique du terme, du système car limpact de la concurrence pour l'Inrap est de plus en plus important. Or il me semble que cette régulation ne peut plus reposer aujourd'hui sur le seul critère scientifique et qu'il faut examiner les conditions sociales, économiques, environnementales d'intervention, mais tout ce qui relève de la politique de prévention, en termes d'hygiène et de sécurité. Il y a urgence!

\section{La recherche et ses méthodes}

JPJ Les avancées méthodologiques se sont

beaucoup faites grâce à une mécanisation de grande qualité et d'une précision extraordinaire. Les systèmes d'information géographique apportent aussi une facilitation des relevés et une nouvelle appréhension de la masse des données collectées. Par ailleurs, nous expérimentons des drones capables de faire des relevés numériques qui permettront de gagner un temps précieux pour ne pas allonger les délais d'intervention. Cela pose le problème récurrent de ce que doit être la prescription de la part de l'État. Il ne faut pas qu'en déterminant des principes méthodologiques, elle muselle les capacités d'innovation en termes de techniques et de méthodes.

JPD Effectivement, la mécanisation a constitué une révolution dans les années 1970 et il ne faut pas que cet élan s'arrête. Ces méthodes ont permis le développement d'une archéologie spatiale et territoriale, l'étude de sites sur de très grandes surfaces qui a transformé complètement la vision de nombreuses périodes. Et dans le domaine du paléo-environnement, le fait de suivre des tracés 
linéaires qui sont des transects à travers le paysage, a permis d'approcher beaucoup plus finement les fluctuations climatiques, les phénomènes de dégradation des sols, des occupations, etc. JPJ L'exposition sur les Gaulois, que nous avons coproduite avec la Cité des sciences et de l'industrie, montre toutes les avancées de la connaissance sur la Gaule, dont on ne connaissait bien que les oppida. Les premières fermes «indigènes » n'ont été découvertes qu'il y a quelques décennies. Dans le même ordre d'idées, dans les dernières années, on a découvert un chapelet de bâtiments paléochrétiens... C'est très important: on met au jour trois ou quatre nouveaux baptistères et cela redessine complètement la mise en place des grands épiscopats à la fin de l'Empire et le développement de la chrétienté au haut Moyen Âge.

\section{L'évolution de la prescription}

JPD Il me semble que l'État doit réfléchir à la notion de prescription parce que de celle-ci découle la recherche.

JPJ C'est d'ailleurs une réflexion d'actualité et le ministère de la Culture s'en est emparé et nous nous en réjouissons. Il faut en effet reposer la question : Qu'est-ce que c'est qu'une prescription? Pourquoi prescrit-on? Où et pour quel objectif? Dans les années 1980, la prescription était fondée certes sur l'intérêt du site, mais aussi sur la solvabilité des aménageurs car le prescripteur était aussi le négociateur. Avec la loi de 2001, puis de 2003, il y a eu une déconnexion totale. Il faut arriver à intégrer tous les résultats des enquêtes nationales à une réflexion plus globale du CNRA pour déterminer des priorités à même d'orienter les choix : on n'a jamais et on ne pourra jamais tout fouiller ! Et puis, il faut se poser la question du contenu de la prescription : si bien entendu une prescription repose sur des objectifs, doit-elle aller jusqu'aux moyens? Je ne le pense pas et la direction générale des patrimoines partage ce sentiment car sinon, la concurrence ne peut se faire que sur le prix, et alors, c'est le «moins disant» qui l'emporte presque toujours. Le ministère de la Culture a ainsi entamé un important travail de clarification auquel je suis particulièrement attentif. Nous devons parvenir à un consensus sur ce sujet entre tous les acteurs, et rapidement au regard des enjeux.

\section{L'avenir de l'Inrap}

JPD L'Inrap connait encore beaucoup de difficultés, mais avec le recul, je suis frappé par les progrès accomplis, en interne en termes de structuration, et en externe vis-à-vis de la communauté scientifique, des aménageurs et du public. Il y a eu un véritable désenclavement de l'archéologie préventive. On le mesure bien à travers le succès de l'exposition de la Cité des sciences ou des Journées de l’Archéologie. S'il y a cette demande sociale, c'est parce que l'archéologie permet aux gens de réfléchir sur leur propre société, en replaçant cette réflexion dans la longue durée, mais encore fallait-il leur donner les outils pour s'approprier cette connaissance. JPJ Pour ma part, je suis frappé par le développement du travail scientifique et par le nombre des publications des archéologues de
l'Inrap. Le rapport entre le temps de recherche et le nombre de publications est extrêmement favorable: trois publications dans des revues de bon niveau pour un équivalent temps plein ; c'est le même ratio que le CNRS. Par ailleurs, nous avons concrétisé le projet de collection scientifique - les deux premiers volumes de «Recherche archéologique» viennent de paraitre et la collection va atteindre le rythme de quatre ouvrages par an - sans évoquer le succès en librairie des collections de très bonne vulgarisation que tu avais lancées. Dans les années à venir, il faut que l'Inrap soit reconnu dans le paysage de la recherche comme un " organisme de recherche » à part entière et qu'il ne soit plus contesté dans le paysage politique, et là, il y a encore beaucoup de progrès à accomplir... C'est une entreprise que tu as commencée, c'est un combat que je poursuis.

JPD Il me semble aussi que l'Inrap doit renforcer la diffusion pour améliorer la perception de l'archéologie par la population et par les aménageurs. Cela étant, le paramètre archéologique est de mieux en mieux pris en compte par les aménageurs, qui ont progressivement intégré les contraintes et les avantages de l'archéologie préventive.

JPJ Une des conditions, c'est la consolidation financière de l'institut. Nous avons beaucoup progressé ces derniers mois avec - enfin ! - la recapitalisation de l'institut. 2012 et 2013 devraient être des années décisives avec le rodage de la nouvelle redevance. Évidemment, si l'Inrap bénéficie d'une meilleure santé financière, nos efforts seront plus orientés sur la recherche et la valorisation que sur la consolidation de la gestion, qui était un préalable essentiel. La signature avec l'État du premier contrat de performance de l'institut pour la période 2011-2013 est, de ce point de vue, une étape essentielle.

JPD Après avoir suscité un important travail collectif, qui a abouti au projet d'établissement de 2004, Nicole Pot avait beaucoup travaillé à un premier contrat de performance, sans parvenir à le signer avec l'État... Ce nouveau contrat marque donc un progrès important. Mais avec cette nouvelle RAP, amoindrie au dernier moment par le parlement, vous vous retrouvez un peu dans la situation de 2002, sans très bien savoir où vous allez financièrement... C'est un peu désolant quà nouveau il manque un petit rien. Ce qui fait que, à chaque fois que le sujet revient au Parlement ou ailleurs, on entend : «Ah, c'est encore l'Inrap!» Pourtant, par rapport début des années 2000, vous n'êtes plus très loin du but.

JPJ En effet, il faut arriver à entrer dans la (relative) quiétude du fonctionnement d'une structure de recherche normale. Mais nous en approchons progressivement. L'Inrap est déjà une référence en matière d'archéologie préventive en Europe et c'est un préalable pour que les hommes politiques nous adoptent définitivement. 


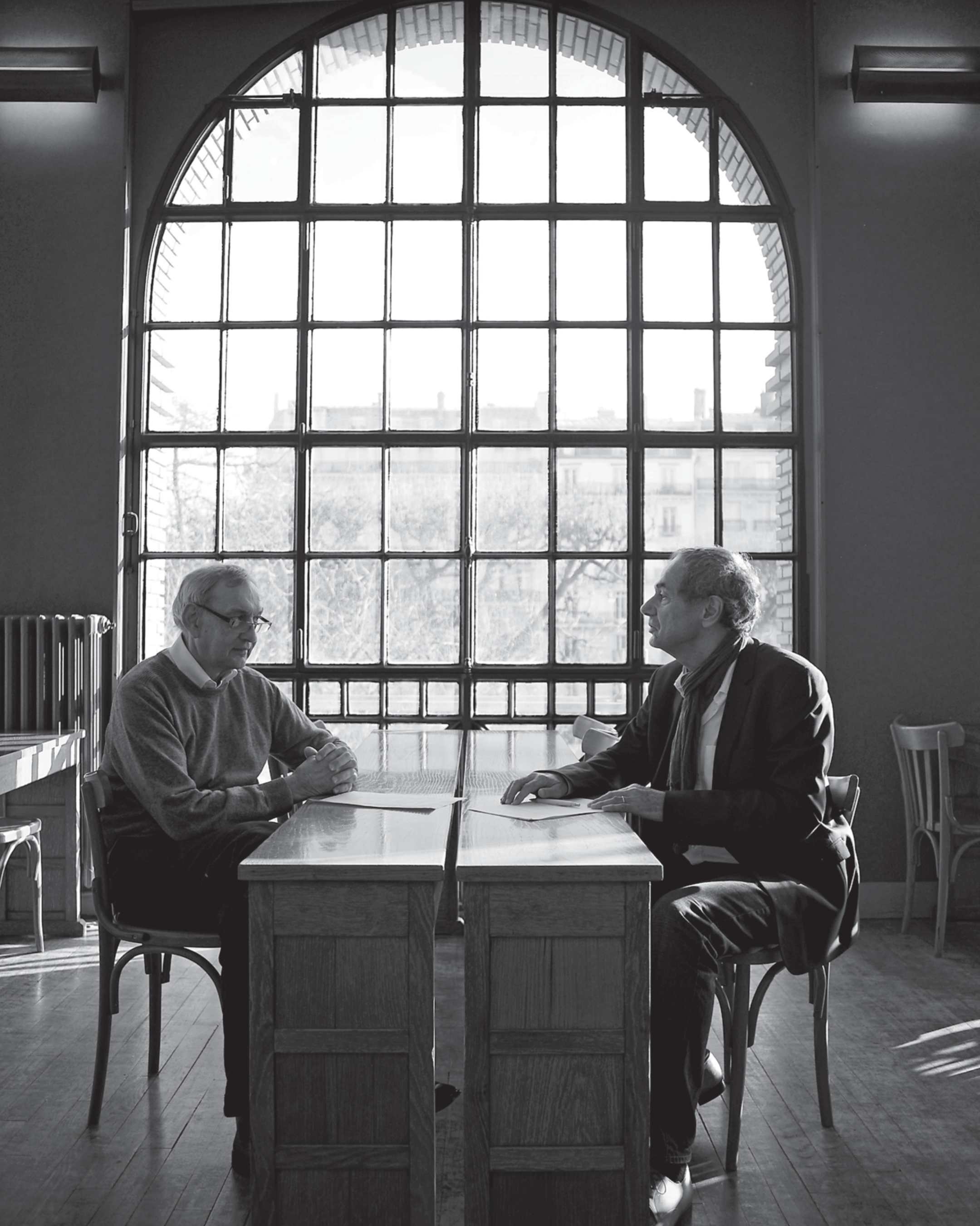

